\title{
Revisão
}

\section{Resistência aos antibióticos de uso oral a partir de isolados de urina de pacientes não hospitalizados}

\author{
Maria Eduarda Campos Bezerra1 (iD Rosilma de Oliveira Araújo Melo² iD Francisco Montenegro de \\ Melo $^{3}$
}

${ }^{1}$ Discente do curso de Biomedicina do Centro Universitário Maurício de Nassau

${ }^{2}$ Co-orientadora, docente do Centro Universitário Maurício de Nassau

${ }^{3}$ Orientador, médico especialista em microbiologia clínica pela Universidade Federal de Pernambuco

$\triangle$

Maria Eduarda Campos Bezerra

Edited by

Juliana Ramos Andrade
Palavras-chave:

Escherichia coli. Infecção

Resistência a antibióticos

Trato urinário

Keywords:

Escherichia coli

Infection

Antibiotic resistance

Urinary tract

\section{Resumo}

O índice da resistência antimicrobiana cresce progressivamente no mundo e acaba limitando a eficácia de tratamento de doenças comuns, como a infecção do trato urinário. Além disso, na atualidade, a resistência antimicrobiana é apontada como um dos principais problemas de saúde pública. Diante do exposto, o objetivo do presente estudo foi avaliar a resistência bacteriana frente aos antibióticos orais mais comumente utilizados para o tratamento das infecções urinárias em pacientes ambulatoriais, bem como a identificação das bactérias isoladas. Foi realizado um estudo observacional experimental com abordagem quantitativa no período de janeiro a maio de 2019, desenvolvido em um hospital privado de alta complexidade localizado na cidade do Recife, PE. Foram incluídas no estudo todas as uroculturas positivas de pacientes adultos atendidos no ambulatório, para a identificação bacteriana, o equipamento Matrix Associated Laser Desorption-lonization - Time of Flight. Foram analisadas um total de 379 amostras positivas de uroculturas, das quais $16(4,2 \%)$ representavam espécies sem interesse clínico, pois obtiveram uma porcentagem de frequência muito baixa, bem como 327 (86,3\%) pertenciam a três espécies de bactérias Gram-negativas: Escherichia coli (72,8\%), Klebsiella pneumoniae (16,2\%) e Proteus mirabilis (1 1\%). Enquanto nas $36(9,5 \%)$ amostras foram identificadas duas espécies Gram-positivas: Staphylococcus saprophyticus (66,7\%) e Enterococcus faecalis (33,3\%). Foi possível observar que o antibiótico oral com maior percentual de resistência foi o sulfametoxazol + trimetoprima com $24,5 \%$ seguido da ciprofloxacina com $22,1 \%$, cefuroxima $(16,7 \%)$, amoxicilina + clavulanato $(13,0 \%)$ e nitrofurantoína $(8,1 \%)$. De acordo com recomendação, é excluído o uso de antibióticos que apresentarem resistência acima de $20 \%$, desta forma os únicos antibióticos por via oral que possui perfil terapêutico adequado para o tratamento empírico dos pacientes adultos com ITU na região do presente estudo são cefuroxima, amoxicilina + clavulanato e nitrofurantoína.

\begin{abstract}
The antimicrobial resistance index grows progressively around the world. It limits the effectiveness of treating common diseases, such as urinary tract infection. In addition, currently, antimicrobial resistance is identified as one of the leading public health problems. Given the above, this study aimed to evaluate bacterial resistance to the most commonly used oral antibiotics for the treatment of urinary infections in outpatients and the identification of isolated bacteria. An experimental, observational study with a quantitative approach was carried out from January to May 2019, developed in a high-complexity private hospital located in Recife, PE. In the study, we use all positive urine cultures from adult patients treated at the outpatient clinic, where the Matrix Associated Laser Desorption-lonization - Time of Flight equipment was used for bacterial identification. A total of 379 positive samples from urine culture samples were analyzed, of which $16(4.2 \%)$ represented species of no clinical interest, as they obtained a very low frequency percentage, as well as 327 (86.3\%) belonged to three species of bacteria Gram-negative: Escherichia coli (72.8\%), Klebsiella pneumoniae (16.2\%) and Proteus mirabilis (11\%). While in the $36(9.5 \%)$ samples, two Gram-positive species were identified: Staphylococcus saprophyticus $(66.7 \%)$ and Enterococcus faecalis (33.3\%). It was observed that the oral antibiotic with the highest percentage of resistance was sulfamethoxazole + trimethoprim with $24.5 \%$ followed by ciprofloxacin with $22.1 \%$, cefuroxime (16.7\%), amoxicillin + clavulanate (13.0\%) and nitrofurantoin (8.1\%). According to the recommendation, antibiotics that present resistance above $20 \%$ are excluded; thus, the only oral antibiotics that have an adequate therapeutic profile for the empirical treatment of adult UTI patients in the present study region are cefuroxime amoxicillin + clavulanate and nitrofurantoin.
\end{abstract}




\section{Introdução}

A resistência antimicrobiana (RAM) neste momento é apontada como um dos principais problemas de saúde pública em decorrência da não responsividades dos tratamentos medicamentosos frente aos patógenos cada vez mais multirresistentes. ${ }^{1,2}$ Isso ocorreu, também, devido ao uso descontrolado e indevido de antibióticos ${ }^{3}$, além disso, pacientes não hospitalizados com infecção urinária têm apresentado alto risco em desenvolver resistência aos antibióticos. ${ }^{4}$

Como consequência da RAM, muitos dos medicamentos geralmente utilizados na terapêutica não apresentam eficácia e as infecções permanecem no hospedeiro tendendo a uma ameaça de proliferação. ${ }^{5} \mathrm{O}$ uso indiscriminado dos antimicrobianos amplia a pressão seletiva e beneficia o progresso da resistência, pois muitos pacientes acabam se automedicando além de não cumprir com o tratamento corretamente. ${ }^{6}$

A infecção do trało urinário (ITU) é uma das infecções mais comuns na prática clínica, seja ambulatorial ou hospitalar e podem atingir todas as idades. ${ }^{78}$ Além disso, é bastante frequente em mulheres devido a uretra ser mais curta e por apresentar certa proximidade com o ânus há uma maior facilidade para a colonização de microrganismos. ${ }^{6}$ Causam desde uma cistite até uma pielonefrite, neste último caso é considerado grave por acometer o parênquima renal. ${ }^{7}$ Dentre os principais sintomas estão a febre, disúria, nictúria, urgência urinária, frequência urinária aumentada e dor lombar e/ou suprapúbica. 6,9,10

As bactérias Gram-negativas são as maiores responsáveis pelas ITU, dentre elas, Escherichia coli, importante uropatógeno de origem intestinal, sendo a causadora em mais de $60 \%$ dos casos. Porém, também são frequentes Klebsiella pneumoniae, Proteus mirabilis e entre as Gram-positivas, destaca-se o Staphylococcus saprophyticus, em $6 \%$ dos casos. ${ }^{6,11}$

A triagem para o diagnóstico da infecção urinária pode ser realizada através do exame de urina tipo 1, mas não é específico como a cultura de urina ${ }^{11}$, padrão-ouro para o diagnóstico laboratorial das ITU ${ }^{8}$ que permite $\circ$ isolamento do agente etiológico. ${ }^{10}$ Além disso, possibilita o estudo da susceptibilidade do microrganismo aos antibióticos.

A decisão adequada por tratamento empírico necessita do conhecimento dos uropatógenos envolvidos e qual o seu perfil de resistência aos antibióticos. ${ }^{12-14}$ Os antimicrobianos receitados mais frequentemente para o tratamento dessas infecções são: sulfametoxazol + trimetoprima, fluoroquinolonas (ciprofloxacina ou nor floxacina), amoxicilina + clavulanato e nitrofurantoína. É fundamental que o profissional da área da saúde considere o perfil de resistência do uropatógeno para um tratamento medicamentoso mais adequado. ${ }^{6}$

O índice da resistência antimicrobiana (RAM) cresce progressivamente no mundo e acaba limitando a eficácia de tratamento de doenças comuns, como a infecção urinária. É frequente, estudos demonstrarem o perfil da RAM de pacientes nosocomiais, sendo assim a ampliação de pesquisas de perfil de resistência de pacientes ambulatoriais é extremamente necessária.

Diante disso, o objetivo foi avaliar a resistência bacteriana frente aos antibióticos orais mais comumente utilizados para o tratamento das ITU em pacientes ambulatoriais, bem como a identificação das bactérias isoladas.

\section{Métodos}

Foi realizado um estudo observacional experimental com abordagem quantitativa no período de janeiro a maio do ano de 2019 e desenvolvido em um hospital privado de alta complexidade, localizado na cidade de Recife, Pernambuco. Foram incluídas no estudo todas as uroculturas positivas de pacientes adultos de ambos os sexos que foram atendidos no ambulatório do referido hospital.

As uroculturas de cada paciente foram inoculadas em placas de meio cromogênico (CHROMRID) com alças calibradas descartáveis que possui capacidade de $0,001 \mathrm{~mL}$ e incubadas por 24 horas a $35^{\circ} \mathrm{C}$. Utilizamos o critério de $\mathrm{Kass}^{15}$ para classificar as amostras em positivas, duvidosas ou negativas e foram selecionadas apenas as amostras positivas.

Após o isolamento primário, foi utilizado, para a identificação bacteriana, o equipamento MALDI - TOF (Matrix Associated Laser Desorption-lonization - Time of Flight) que mede proteínas altamente abundantes que são encontradas em todos os microrganismos. Os padrões característicos dessas proteínas são utilizados para identificar de forma confiável e precisa um microrganismo específico. ${ }^{16,17}$

Em seguida, foi utilizado o PHOENIX-BD, um aparelho de automação que tem a capacidade de determinar a susceptibilidade aos antibióticos. Esse aparelho possui painéis individuais específicos para urocultura que contém um poço em cada painel com diversos antibióticos em diferentes concentrações. Entretanto, 
não foi testada a fosfomicina, pois o equipamento não disponibiliza. Foi inserida em cada painel uma suspensão bacteriana de algumas colônias de uma cultura após terem sido homogeneizadas em um vortex e a turbidez ajustada a 0,5 da escala Mcfarland. ${ }^{18}$ Em seguida, o painel foi incubado no aparelho PHOENIX-BD a $35^{\circ} \mathrm{C}$ e aguardado a finalização do processo.

Os dados foram analisados utilizando-se o programa Excel e Microsoft Office versão 365 $R$ por gráficos e tabelas.

\section{Resultados}

Foram analisadas um total de 379 cepas (Figura 1), onde desse montante $327(86,3 \%)$ pertenciam a três espécies de bactérias Gram-negativas: Escherichia coli $(72,8 \%)$, Klebsiella pneumoniae $(16,2 \%)$ e Proteus mirabilis (11\%). Enquanto nas demais 36 (9,5\%) amostras foram identificadas duas espécies Grampositivas: Staphylococcus saprophyticus $(66,7 \%)$ e Enterococcus faecalis (33,3\%) (Figura 2). Demais bactérias foram responsáveis por $4,2 \%$ das cepas de um total de 16 amostras, além disso, sem interesse clínico para o presente estudo pois apresentaram uma porcentagem de frequência muito baixo.

Figura 1: Classificação geral das bactérias isoladas de uroculturas positivas analisadas de pacientes ambulatoriais.

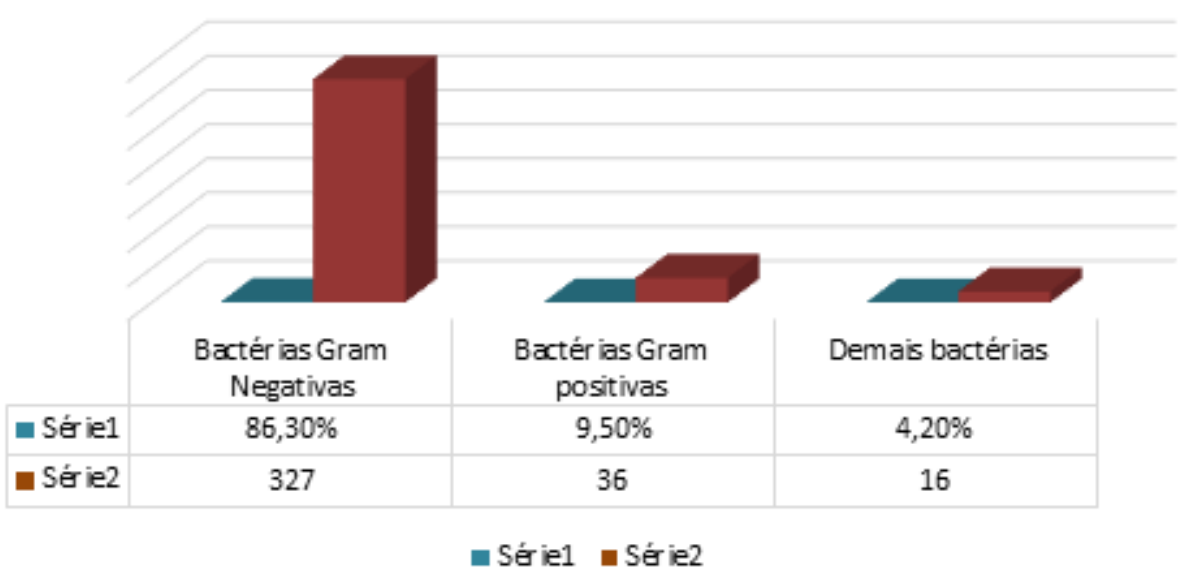

Figura 2. Identificação das principais espécies bacterianas isoladas de pacientes ambulatoriais.

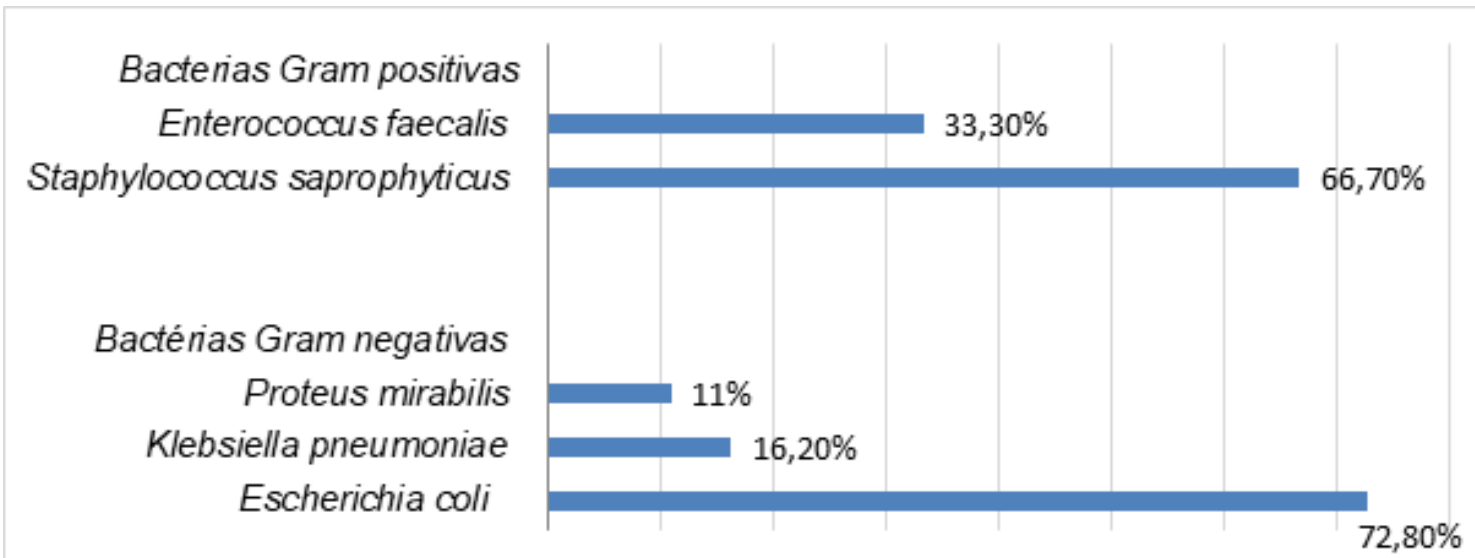


As porcentagens de resistência aos antibióticos de uso oral de acordo com o total de cada microrganismo isolado estão representadas na Tabela 1 , sendo possivel notar que a $\mathrm{E}$. coli se mostrou $40,3 \%$ resistente ao sulfametoxazol + trimetoprima e $26,5 \%$ para a ciprofloxacina. Entretanto, o E. faecalis, apresentou $35,0 \%$ e 33,3\% de resistência a ciprofloxacina e sulfametoxazol + trimetoprima, respectivamente.

Tabela 1. Porcentagem de resistência aos antibióticos de uso oral de acordo com o total de amostras de cada microrganismo isolado.

\begin{tabular}{|c|c|c|c|c|c|}
\hline \multirow[t]{2}{*}{ Antibiótico } & \multicolumn{5}{|c|}{ Microrganismo } \\
\hline & $\begin{array}{l}E_{\text {coli }} \\
\text { coli }\end{array}$ & $\begin{array}{c}\mathrm{K} . \\
\text { pneumoniae }\end{array}$ & $\underset{\text { mirabilis }}{P}$ & $\underset{\text { saprophyticus }}{\text { S. }}$ & $\underset{\text { faecalis }}{E}$ \\
\hline $\begin{array}{l}\text { Amoxicilina + } \\
\text { clavulanato }\end{array}$ & $4,2 \%$ & $26,4 \%$ & $11,1 \%$ & \# & $10,6 \%$ \\
\hline Cefuroxima & $5,4 \%$ & $22,6 \%$ & $22,3 \%$ & \# & $\#^{*}$ \\
\hline Ciprofloxacina & $26,5 \%$ & $22,6 \%$ & $22,3 \%$ & $4,2 \%$ & $35,0 \%$ \\
\hline Nitrofurantoína & $1,6 \%$ & $1,5 \%$ & $100 \% *$ & $4,2 \%$ & $25,0 \%$ \\
\hline $\begin{array}{l}\text { Sulfametoxazol } \\
+ \text { trimetoprima }\end{array}$ & $40,3 \%$ & $31,0 \%$ & $13,9 \%$ & $4,2 \%$ & $33,3 \%$ \\
\hline
\end{tabular}

O antibiótico oral com maior percentual de resistência foi o sulfametoxazol + trimetoprima com $24,5 \%$ seguido da ciprofloxacina com $22,1 \%$, cefuroxima (16,7\%), amoxicilina + clavulanato $(13,0 \%)$ e nitrofurantoína $(8,1 \%)$, observado na figura 3 .

Figura 3. Porcentagem de resistência aos antibióticos de uso oral mais utilizados no tratamento de ITU.

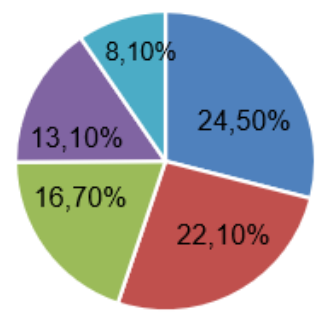
- Sulfametoxazol + trimetoprima = Ciprofloxacina
- Cefuroxima
- Amoxicilina + clavulanato
- Nitrofurantoína

\section{Discussão}

E. coli é o patógeno mais frequente nas ITU devido ao fato de ser um microrganismo pertencente a microbiota normal do intestino que se adere ao cólon, por exemplo e coloniza através de suas fímbrias e adesinas, dificultando assim, sua eliminação através do fluxo urinário. ${ }^{12,15,19,20}$ Dado confirmado pelo predomínio de $62,8 \%$ no presente estudo, bem como semelhante a outras pesquisas. ${ }^{3,15,19}$ Além disso, a prevalência das estirpes encontradas nesse estudo corrobora as descritas na literatura, sobretudo em relação às três mais frequentes: E. coli, K. pneumoniae e Proteus mirabilis3,12,19 Outros estudos, realizado na cidade de Florianópolis e em Minas Gerais também apontaram o predomínio de bactérias Gram-negativas, apresentando porcentagem de 93,8 e 94,1 respectivamente. ${ }^{19,21}$

Dentre as bactérias Gram-positivas, aquelas da família Micrococcaceae principalmente os cocos, são os pató$\neg$ genos isolados com maior frequência. As infecções causadas por esse grupo bacteriano podem decorrer a partir de multiplicação local ou sistêmica. Além disso, o principal gênero relacionado a esta família é o Staphylococcus onde a frequência foi confirmada por dois estudos realizados em Goiás ${ }^{22,23}$ e um em São Paulo6, além do presente estudo. Tem se observado uma maior frequência destes microrganismos associados a doenças adquiridas em comunidades, principalmente em infecções do trato urinário. ${ }^{23}$

A susceptibilidade de uropatógenos à sulfametoxazol + trimetoprima no Brasil é de 50,6\% ${ }^{24}$ dado que não é semelhante ao que foi visto no presente estudo, porém há uma recomendação que cita que em áreas nas quais a resistência local for maior que $20 \%$ não é recomendada a antibioticoterapia com o este medicamento ${ }^{25} \mathrm{e}$ o presente estudo demonstrou porcentagem de resistência total de 24,5. Esses dados confirmam, então, que segundo o estudo realizado na cidade de Florianópolis, o sulfametoxazol + trimetoprima não deve ser utilizado empiricamente. ${ }^{19}$

O segundo antimicrobiano com maior porcentagem de resistência na presente pesquisa foi a ciprofloxacina, que apresentou 22,1\%. Além disso, em relação aos microrganismos resistentes houve uma maior frequência de E. coli $(26,5 \%)$. Um estudo nacional ${ }^{19}$ apresentou porcentagem de 22,7 e Reis ${ }^{6}$ apontou 22,4\%. Diante disso, foi visto que o presente estudo possui uma taxa de aumento de 16,6 e 18,3\% respectivamente, na qual reflete, provavelmente, o uso generalizado e indevido deste antimicrobiano ao longo do tempo para o tratamento de ITU, sendo compreensível a não recomendação para um tratamento empírico.

\section{Conclusão}

O surgimento de resistência bacteriana aos antimicrobianos é inevitável, em virtude da evolução natural das espécies. Como o tratamento para ITU em sua grande maioria é empírico, acaba contribuindo para o desenvolvimento de resistência aos principais patógenos e antimicrobianos corriqueiramente utilizados. Entretanto, o controle na sua utilização pode limitar o aparecimento de linhagens multirresistentes. 
Baseado na recomendação referenciada sobre o uso do antibiótico com percentual inferior a $20 \%$ deve ser usado para tratamento, desta forma os únicos antibióticos por via oral com perfil terapêutico adequado para o tratamento empírico dos pacientes adultos com ITU no Recife são: cefuroxima, amoxicilina + clavulanato e nitrofurantoína. Além disso, como a porcentagem de resistência a ciprofloxacina é semelhante ao sulfametoxazol + trimetoprima, também não seria indicado o uso desse antibiótico para tratamento empírico.

Sendo necessária a conscientização deste problema mundial, além de medidas para evitá-lo, como por exemplo: o uso racional dos antibióticos e prevenção de infecções bacterianas são essenciais. Bem como a realização de estudos com variação em diversas regiões sobre a resistência bacteriana para uma melhor epidemiologia da antibioticoterapia.

Maria Eduarda Campos Bezerra

https://orcid.org/0000-0003-2773-279X

Rosilma de Oliveira Araújo Melo

https://orcid.org/

Francisco Montenegro de Melo

https://orcid.org/0000-0001-6690-6823

\section{Referências}

1. Loureiro RJ, Roque F, Teixeira Rodrigues A, Herdeiro MT and Ramalheira E. $O$ uso de antibióticos e as resistências bacterianas: breves notas sobre a sua evolução. Rev Port Saúde Pública 2016;34(1):77-84 Doi:10.1016/i. rpsp.2015.11.003

2. Kranz J, Schmidt S, Lebert C, Schneidewind L, Schmiemann $G$ and Wagenlehner F. Uncomplicated Bacterial Community-Acquired Urinary Tract Infection in Adults. Dtsch Arztebl Int 2017; 1 14(50):866-873 Doi:10.3238/ arztebl.2017.0866

3. Carvalho T, Nogueira J, Trigo I, Oliveira C and Melo L. Avaliação dos Agentes Isolados e Perfil de Sensibilidade aos Antibióticos nas Uroculturas Efectuadas na Área de Influência e Região Envolvente da USF da Barrinha - Esmoriz. Rev Port Med Geral Fam 2019;35:361-366 Doi:10.32385/rpmgf.v35i5.12188

4. Mohamed MA, Abdifetah O, Hussein FA and Karie SA. Antibiotic resistance pattern of Escherichia coli isolates from outpatients with urinary tract infections in Somalia. J Infect Dev Ctries 2020; 14(3):284-289 Doi:10.3855/jidc. 12189

5. Antimicrobial resistance [Internet]. World Health Organization; Available from: https://www.who.int/health-topics/ antimicrobial-resistance.

6. Reis AC, Santos SR, Souza SC, Saldanha MG, Pitanga TN and Oliveira RR. Ciproflacin Resistance Pattern Among Bacteria isolated from patients with community-acquired urinary urinary tract infection. Rev Inst Med Trop Sao Paulo 2016;58(53): 1-6 Doi:10.1590/s1678-9946201658053

7. Manual - Definição dos Critérios Nacionais de Infecções Relacionadas à Assistência à Saúde - Infecção do Trato Urinário (ITU) Agência Nacional de Vigilância Sanitária; Available from: www.anvisa.gov.br/servicosaude/microbiologia/mod 12004.pdf.

8. Dias IOV, Coelho AM and Dorigon I. Infecção do trato urinário em pacientes ambulatoriais: prevalência e perfil de sensibilidade aos antimicrobianos em estudo realizado de 2009 a 2012 Saúde (Santa Maria) 2015;41(1):209. 218 Doi: 10.5902/2236583415455

9. Gbinigie OA, Ordóñez-Mena JM, Fanshawe TR, Plüddemann $A$ and Heneghan $C$. Diagnostic value of symptoms and signs for identifying urinary tract infection in older adult outpatients: Systematic review and meta-analysis. J Infect 2018;77(5):379-390 Doi:10.1016/i. jinf.2018.06.012

10. Freitas RB, Resende JA, Mendonça BG, Antonio T, Fortunato RS and Oliveira MACA. Infecções do trato urinário de origem hospitalar e comunitária: revisão dos principais micro-organismos causadores e perfil de susceptibilidade. Rev Científica Fagoc 2016; 1 (1):55-62

11. Oliveira SM and Santos LLG. Infecção do trato urinário: estudo epidemiológico em prontuários laboratoriais. J Health NPEPS 2018;3(1):198-210

12. Costa MC, Pereira PM, Bolotinha C, Ferreira A, Cardoso R, Monteiro $C, \ldots$ Gomes JF. Frequência e Susceptibilidade Bacteriana em Infecções Urinárias -dados de um laboratório de Lisbo. Parte II. Rev Lus Ciências Saúde 1(6):87-103

13. Bertoni G, Pessacq P, Guerrini MG, Calmaggi A, Barberis $F$, Jorge $L$, . . Mykietiuk A. Etiología y resistencia a antimicrobianos de la infección no complicada del tracto urinario. Medicina (Buenos Aires) 2017;77(4):304-308

14. Gajdács $M$, Ábrók M, Lázár A and Burián K. Comparative Epidemiology and Resistance Trends of Common Urinary Pathogens in a Tertiary-Care Hospital: A 10-Year Surveillance Study. Medicina (Kaunas) 2019;55(7):1-15 Doi: 10.3390/medicina55070356

15. Machado AD, Naumann DC, Ferrazza MHSH, Tenfen A, Silva BYG and Webe KJRbac. Prevalência de infecção urinária em um laboratório de análisesclínicas da cidade de Jaraguá do Sul, SC, no ano de 2017. Rev Bras Anal Clin 2019;51(13):213-218 Doi:10.21877/2448 3877.201900821

16. Microbial Identification for Clinical Laboratories (US-IVD) [Internet]. Bruker; Available from: https://www.bruker. com/en/products-and-solutions/microbiology-and-diagnostics/microbial-identification-for-clinical-laboratories-us-ivd.html.

17. Pasternak J. New methods of microbiological identification using MALDI-TOF. Einstein (Sao Paulo) 2012;10(1):1 18 119 Doi:10.1590/s 1679-45082012000100026

18. Padrão de turvação preparado BLL: BD; Available from: https://www.bd.com/resource.aspx?IDX=20546. 
19. Alves DMD, Edelweiss MK and Botelho LJ. Infecções comunitárias do trato urinário: prevalência e susceptibilidade aos antimicrobianos na cidade de Florianópolis. Rev Bras Med Fam Com 2016;11(38):1-12 Doi:10.5712/rbmfc 11 (38) 1187

20. Heilberg IP and Schor N. Diagnosis and clinical management of urinary tract infection. Rev Assoc Med Bras (1992) 2003;49(1):109-116 Doi:10.1590/s010442302003000100043

21. Júnior GES, Guerra KDS, D'Angelis CEM, Veloso DA and Crisóstomo CM. Infecções do trato urinário: frequência e etiologia em pacientes não hospitalizados. Rev Unimontes Cient 2018;20(1):113-126

22. Castro BG, Pinto LS and Souto RCF. Prevalência de bactérias Gram-positivas em infecção do trato urinário. Rev
23. Bras Anal Clin 2019;51(4):322-327 Doi:10.21877/2448 3877.201900791

24. Santos LS, Damasceno NdS and Souto RCF. Resistência de bactérias Gram-positivas isoladas de infecção do trato urinário no LAC/PUC Goiás Rev Bras Anal Clin 2019;51(2): 143148 Doi:10.21877/2448-3877.201900741

25. Chávez D, A J, Castillo P, R A, Alcocer Q, Flores G, ... E D. Resistencia y sensibilidad bacteriana en urocultivos en una población de mujeres de ecuador. Rev Med 2018;26(2):22-28

26. Pires MCS, Frota KS, Junior POM, Correia AF, Cortez-Escalante JJ and Silveira CA. Prevalência e suscetibilidades bacterianas das infecções comunitárias do trato urinário, em Hospital Universitário de Brasília, no período de 2001 a 2005. Rev Soc Bras Med Trop 2007;40(6):Doi:101590/ S0037-86822007000600009 